Proceedings of the 2006 Winter Simulation Conference

L. F. Perrone, F. P. Wieland, J. Liu, B. G. Lawson, D. M. Nicol, and R. M. Fujimoto, eds.

\title{
QUALITATIVE SIMULATION FOR MANAGER SELECTION DECISION-MAKING BASED ON MANAGERIAL SELF-EFFICACY
}

\author{
Hu Bin \\ School of Management \\ Huazhong University of Science and Technology \\ Wuhan, 430074, P.R.CHINA
}

\author{
Dong Shengping \\ School of Management \\ Huazhong University of Science and Technology \\ Wuhan, 430074, P.R.CHINA
}

\begin{abstract}
To explore the dynamics of managerial self-efficacy, a causality model is proposed based on the theory of Bandura's Self-efficacy and Human Resource Management. Furthermore, a qualitative simulation model is built and a simulation algorithm is designed. The simulation is achieved using Visual Basic 6.0. An example of application is illustrated. Simulation results show that the proposed method can be served as a decision making tool on manager selection for enterprise.
\end{abstract}

\section{INTRODUCTION}

Self-efficacy is defined as "beliefs in one's capacity to organize and execute the courses of action required to produce given attainments" (Bandura 1997). It means perception of one's ability to plan, control and take action to reach a particular goal. Bandura (1997) argued that forming and developing individual self-efficacy was affected by four factors: enactive mastery experiences, vicarious experiences, verbal persuasion, physiological and affective states. Bandura and Wood (1989) pointed out that managers' managerial self-efficacy was related to outlook on ability or tendency to target, controllable degree perceived, etc. Furthermore, it is found that managerial self-efficacy and objective setting have close causal relation. In addition, the active feedback exerts significant effect on a team's selfefficacy (Earley 1999).

To explore the interaction between psychological factors related to self-efficacy in team management, the term of Managerial Self-efficacy (MSE) is thus used to define managers' judgments of their capability to manage his team in diverse situations. The experiment indicated that MSE (Managerial Self-efficacy) of managers had highly positive effect on their organizational working performance (Bandura 1989; Wood 1989; Cervone 1991). MSE of managers is one of the most important variables affecting ones' managerial performance (Robertson 1993), and it was also related remarkably to the subordinated team's working performance (Lauschruger 1994).

Due to high predictability of MSE to managerial working performance, it is effective to select a candidate for a key managerial position of enterprise by measuring one's MSE. This candidate maybe yield high performance on his position with most probability. Different from actual experiments (Wood and Bandura 1989), computer simulation is used in the present research to depict the change process of MSE, which will help for decisionmaking in manager selection.

Earlier applications of computer simulation to management field were in operation level rather than in decision-making level in enterprise. One of the reasons is that there are many subject factors and other complex factors involved in decision-making level. However, there are much more human factors involved in selection decision process, and self-efficacy is from the domain of social psychology. These make quantitative simulation much difficult for aiding selection based on self-efficacy. Thus, qualitative simulation is employed to solve the problem.

As one of qualitative simulation methods, QSIM (Qualitative SIMulation) developed by Kuipers (1986) is widely accepted. It is designed to explain and predict behaviors of physical systems (Kuipers 1993; Clancy and Kuipers 1989). QSIM runs in this way: the state of system is evolved from the initial state. All subsequent states are generated by the state transition rules. At each transition, the states that do not meet constraint equations are filtered out. The remained states constitute a new combination of states. The evolution continues from one state in the combination. The above process repeated to generate behaviors of system (Kuipers 1993). QSIM is actually a constraintsatisfied process. Behaviors of system are generated from state variables' transition by rules and be filtered by constraints. Therefore, the mechanism of QSIM runs in the pattern of generating-filtering. However, there are much uncertain and incomplete information on decision-making in management field. QSIM should be improved to explore the interaction dynamically changing along with environ- 


\section{Bin and Shengping}

ment among people. Hu and Xia (2003, 2005) summarized the constraining-generating-filtrating pattern from QSIM and explored its application to marketing and human resource management. Basing on their researches, quantitative simulation characterized by probability is introduced into qualitative simulation. Thus, a modified QSIM, namely QSIM with constraining-generating-filtrating pattern, is proposed to solve the decision-making problems of managers selection based on managerial self-efficacy. An example is used to illustrate the application of the proposed method.

\section{A CAUSALITY MODEL OF MANAGERIAL SELF-EFFICACY}

Based on the above researches and common senses in management field, a causality model of managerial selfefficacy is built as shown as Figure 1.

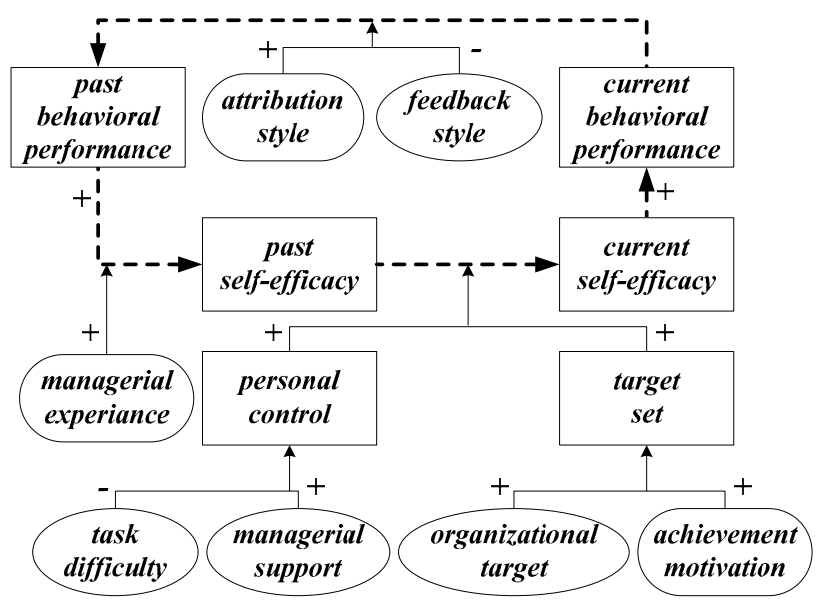

Figure 1: The Causality Model of Managerial Self-Efficacy

In Figure 1, the italic texts enclosed in different frames such as rectangular frames, round rectangular frames and elliptical frames denote different types of variables. Real or broken lines with arrow at the end describe the effects of one variable acting on another. "+" and "-" on the lines means positive and negative effects, respectively. The meanings of variables in Figure 1 are detailed as follows.

Managerial experience $(M E)$ is the managers' experience accumulated during their past managerial activities. It includes the experiences from managers' own or from others' experiences and performances.

Achievement motivation $(A M)$ is the internal motivation for the manager to pursue success in doing his/her task. It comprises two kinds of stable tendency, i.e., tendency for pursuing success and tendency for avoiding failure.
There are two types of self-efficacy, namely past selfefficacy $\left(S E^{\prime}\right)$ and current self-efficacy $(S E) . S E$ ' is the managerial self-efficacy formed before a certain managerial activity beginning. It will transfer to $S E$. $S E$ is the managerial self-efficacy during the activity.

Task difficulty $(T D)$ is the degree of how difficultly a managerial task is completed due to its complexity or uncertainty.

Personal control $(P C)$ means a manager's two personal senses. One sense is that, he/she can feel the degree he/she affects the result of managerial activities. The other sense is that, he/she can feel the degree he/she control the process of managerial activities.

Target set $(T S)$ means the choice or promise made by a manager according to organizational target and individual target.

Organizational target $(O T)$ means the target specified for the managerial activities by organization. It is the standard of final performance evaluation.

Behavioral performance is the factors that have implicit effect on the result of tasks. They do not directly show as the result of tasks. In the proposed model, behavioral performance appears as two types: past behavioral performance $\left(B P^{\prime}\right)$ and current behavioral performance $(B P)$. Different from $B P, B P^{\prime}$ is the behavioral performance exiting before a certain managerial activity beginning. $B P$ is the behavioral performance in the activity.

Feedback style $(F S)$ is the superior's assessment of the performance of managerial activity. It includes active feedback and positive feedback.

Attribution style $(A S)$ presents whether manager attributes his/her performance to stable factors or unstable factors. Stable factor, for example, is his/her ability. Unstable factors include luck, effort and difficulty of task.

Managerial support $(M S)$ is the support of information, resource and others. It comes from manager's superior or colleagues. For example, the power to seize organizational resource authorized by superior, the degree of caring about the task from superior.

The model in Figure 1 comprises three processes:

1. The initialization of $S E$ '. Before beginning a new managerial task, $S E$ ' is yielded by the combination of $B P^{\prime}$ and $M E$.

2. The transition from $S E$ ' to $S E$. After a new managerial task begins, $T D$ and $M S$ will affect a manager's $P C$ directly. The manager's $T S$ comes from his/her $A M$. To satisfy $O T$ given by the organization, one's final target set will be the balance between $T S$ and $O T$. $P C$ and $T S$ of a manager will exert effect on $S E$ ', and $S E$ is then formed. $S E$ changes continuously during the managerial activity. It affects the final $B P$.

3. The change from $B P$ to $B P$ '. Near the end of the activity, the final $B P$ turns into $B P^{\prime}$ with the influ- 


\section{Bin and Shengping}

ence by $F S$ from the superior and one's own $A S$. $B P^{\prime}$ will appear in the next stage.

Thus, the three above processes will run one by one till a new managerial task occurs.

\section{SIMULATION MODEL BASED ON QSIM MODIFIED}

The follows are the details of building a simulation model by integrating the modified QSIM with the causality model of MSE (Figure 1).

\subsection{Knowledge Description}

Variables in the model are divided into two types according to their roles in simulation.

1. State variables. They describe the key characteristics of managers during simulation, and change dynamically with the simulation runs. They are $S E, S E$ ', $B P, B P^{\prime}, P C$ and $T S$, which are enclosed in rectangular frames in Figure 1.

2. External variables. They can be measured or specified by user before the simulation runs, and can be controlled during the simulation runs. External variables are divided into two sub-types further:

(a) Individual variables. They denote properties of individual manager, which have effect on state variables. They are $A S, A M$ and $M E$, which are enclosed in round rectangular frames in Figure 1.

(b) Contextual variables. They denote properties of organizational environment related to organizational background, external environment, managerial tasks, etc. They are subjected to stochastic distribution during the simulation runs. They are $F S, T D, M S$ and $O T$, which are enclosed in elliptical frames in Figure 1.

Qualitative states of the variables defined above at time stage $t_{i}$ are described as dualistic group:

$$
Q S\left(X, t_{i}\right)=(q \operatorname{val} X, q \operatorname{dir} X)
$$

qvalX is the qualitative value of $X$ and qdir $X$ is the change direction here. Let qdir $X$ be "+", "0" and "-", which mean increase, no change and decrease, respectively. qdir $X$ can also be " $2+$ " or " $2-$ " that means increase or decrease with higher degree than "+" or "-".

Changes of variables' qualitative state at all time stages are described as collection of dualistic groups:

$$
Q S(X)=\left(Q S\left(X, t_{0}\right), Q S\left(X, t_{1}\right), \cdots, Q S\left(X, t_{n}\right)\right)
$$

A fuzzy membership function shown as Figure 2 is applied to description of qvalX. Where qvalX $\in$ \{lowest, lower, middle, higher, highest\}. In simulation model, qualitative values are numbered. Let "lowest", "lower", "middle", "higher" and "highest" be 1, 2, 3, 4, 5, respectively. Thus, qualX $\in\{1,2,3,4,5\}$.

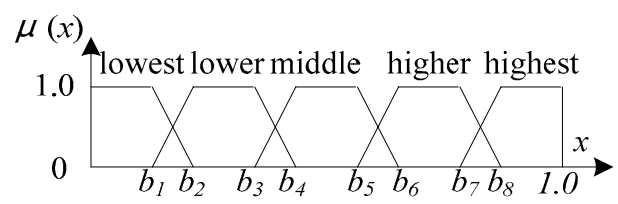

Figure 2: The Fuzzy Membership Function of Variables

The functions are defined as:

$$
\begin{aligned}
& \mu_{1}(x)=\left\{\begin{array}{l}
1 .\left(0<x \leq b_{1}\right) \\
\frac{b_{2}-x}{b_{2}-b_{1}} .\left(b_{1}<x \leq b_{2}\right)
\end{array}\right. \\
& \mu_{i+1}(x)=\left\{\begin{array}{l}
\frac{x-b_{i}}{b_{i+1}-b_{i}} \cdot\left(b_{i}<x \leq b_{i+1}\right) \\
\frac{b_{i+3}-x}{b_{i+3}-b_{i+2}} .\left(b_{i+2}<x \leq b_{i+3}\right)
\end{array} \quad(i=1,2,3)\right. \\
& \mu_{5}(x)=\left\{\begin{array}{l}
\frac{b_{8}-x}{b_{8}-b_{7}} \cdot\left(b_{7}<x \leq b_{8}\right) \\
1 .\left(b_{8}<x \leq 1\right)
\end{array}\right.
\end{aligned}
$$

where $b_{i}(i=1, \ldots, 8)$ are constants. In this paper, let $b_{1}$ $=0.15, b_{2}=0.2, b_{3}=0.35, b_{4}=0.4, b_{5}=0.55, b_{6}=0.6, b_{7}=0.75$, $b_{8}=0.8$. qualX is then specified as followings:

1. If $X$ is a state variable, qual $X$ will be initiated by expert evaluation and the corresponding membership. The membership is calculated by the equation $3,4,5$.

2. If $X$ is contextual variable, qval $X$ will be initiated by the random number within $[0,1.0]$ and the corresponding membership. The membership is calculated by the equation $3,4,5$.

3. If $X$ is individual variables, qval $X$ will be initiated by the measurement of individual properties and the corresponding memberships. The memberships are calculated by the equation $3,4,5$..

The state transition rules are designed basing on Itransition rules and P-transition rules of QSIM (Kuipers 1993). Qualitative value and change direction of variables can be transferred by the state transition rules as follows: 
1. If $q \operatorname{dir} X=+$ and $q \operatorname{val} X\left(t_{i-1}, t_{i-1}\right)<5$, then $q \operatorname{val} X\left(t_{i}\right.$, $\left.t_{i+1}\right)=q \operatorname{val} X\left(t_{i-1}, t_{i}\right)+1$.

2. If $q \operatorname{dir} X=+$ and $q \operatorname{valX}\left(t_{i-1}, t_{i}\right)=5$, then $q \operatorname{valX}\left(t_{i}\right.$, $\left.t_{i+1}\right)=q \operatorname{val} X\left(t_{i-1}, t_{i}\right)$.

3. If qdirX $=$ - and $q \operatorname{val} X\left(t_{i-1}, t_{i}\right)>5$, then $q \operatorname{valX}\left(t_{i}\right.$, $\left.t_{i+1}\right)=q \operatorname{val} X\left(t_{i-1}, t_{i}\right)-1$

4. If $q \operatorname{dir} X=-$ and $q \operatorname{val} X\left(t_{i-1}, t_{i}\right)>1$, then $\operatorname{qvalX}\left(t_{i}\right.$, $\left.t_{i+1}\right)=q \operatorname{val} X\left(t_{i-1}, t_{i}\right)-1$.

5. If $q \operatorname{dir} X=-$ and $q \operatorname{val} X\left(t_{i-1}, t_{i}\right)=1$, then $\operatorname{qvalX}\left(t_{i}\right.$, $\left.t_{i+1}\right)=q \operatorname{valX}\left(t_{i-1}, t_{i}\right)$.

6. If $q \operatorname{dir} X=0$ and $q \operatorname{val} X\left(t_{i-1}, t_{i}\right)=$ any (namely any value), then qvalX $\left(t_{i}, t_{i+1}\right)=q \operatorname{val} X\left(t_{i-1}, t_{i}\right)$.

The changes of contextual variables during simulation runs may lead to various successor behaviors of state variables (i.e., qualitative values and change directions). With simulation advancing, the combination explosion of successor behaviors is yielded. To avoid the combination explosion, the unreasonable successor behaviors should be filtered out according to their probability. The successor behavior with the highest probability will be selected as the most probable qualitative state. The others are filtered out.

To aid selection decision making, A candidate's $S E$ ' is evaluated according to $S E$ 's qualitative values and change directions at all time stages. The best one of the candidates is selected according to the evaluation rules:

1. The highest value rule. After the simulation ends, the candidate who has the highest value of $S E$ is selected.

2. The highest probability rule. After the simulation ends, the candidate who has the highest probability of $S E$ is selected.

3. The combination rule. After the simulation ends, the candidate who has the highest value of $S E$ and also the highest probability of $S E$.

\subsection{Constraint Rules}

To describe the causal relations among variables in the causality model (Figure 1), constraint rules are defined as follows.

1. $\mathrm{SC}+$ constraint rule. $\mathrm{SC}+(X, Y)$ is a dualistic predication indicating the relation of variable $X$ to variable $Y$. The rules are defined as follows:

(a) If $q \operatorname{dir} X\left(t_{i}\right)=+$, then $\operatorname{qdir} Y\left(t_{i+1}\right)=+$

(b) If $q \operatorname{dir} X\left(t_{i}\right)=-$, then $q \operatorname{dir} Y\left(t_{i+1}\right)=-$

(c) If $q \operatorname{dir} X\left(t_{i}\right)=0$, then $q \operatorname{dir} Y\left(t_{i+1}\right)=$ any (i.e., any direction)

2. $\mathrm{SC}$ - constraint rule. $\mathrm{SC}-(X, Y)$ is a dualistic predication indicating the relation of variable $X$ to variable $Y$. The rules are defined as follows:

(a) If $q \operatorname{dir} X\left(t_{i}\right)=+$, then $q \operatorname{dir} Y\left(t_{i+1}\right)=-$

(b) If $q \operatorname{dir} X\left(t_{i}\right)=-$, then $q \operatorname{dir} Y\left(t_{i+1}\right)=+$ (c) If $\operatorname{qdir} X\left(t_{i}\right)=0$, then $q \operatorname{dir} Y\left(t_{i+1}\right)=$ any (i.e., any direction)

3. SC? constraint rule. $\mathrm{SC}$ ? $((X, Y), Z)$ is a ternary predication indicating the relation of variable $X$ and $Y$ to variable $Z$. This rule is defined in Table 1.

According to the above rules, all constraints involved in the model (see Figure 1) are listed in Table 2.

Table 1: The Definition of SC? $((X, Y), Z)$

\begin{tabular}{|c|c|c|}
\hline \multicolumn{2}{|c|}{ If } & Then \\
\hline$q \operatorname{dir} X\left(t_{i}\right)$ & $q \operatorname{dir} Y\left(t_{i}\right)$ & $q \operatorname{dir} Z\left(t_{i+1}\right)$ \\
\hline \multirow{3}{*}{+} & + & + \\
\hline & 0 & + \\
\hline & - &,,+- 0 \\
\hline \multirow{3}{*}{0} & + & + \\
\hline & 0 & 0 \\
\hline & - & - \\
\hline \multirow{3}{*}{-} & + &,,+- 0 \\
\hline & 0 & - \\
\hline & - & - \\
\hline
\end{tabular}

Table 2: All Constraints Involved in the Model

\begin{tabular}{|c|c|c|}
\hline Process & Type & Constraint \\
\hline \multirow{4}{*}{1} & $\mathrm{SC}+(X, Y)$ & $\mathrm{SC}+\left(B P^{\prime}, S E^{\prime}\right), \mathrm{SC}+\left(M E, S E^{\prime}\right)$ \\
\cline { 2 - 3 } & $\mathrm{SC}-(X, Y)$ & none \\
\cline { 2 - 3 } & $\mathrm{SC} ?(X, Y, Z)$ & $\mathrm{SC} ?\left(B P^{\prime}, M E, S E^{\prime}\right)$ \\
\hline \multirow{4}{*}{2} & $\mathrm{SC}+(X, Y)$ & $\begin{array}{r}\mathrm{SC}+(A M, T S), \mathrm{SC}+(O T, T S), \mathrm{SC}+(M S, P C), \\
\mathrm{SC}+(P C, S E), \mathrm{SC}+(T S, S E)\end{array}$ \\
\cline { 2 - 3 } & $\mathrm{SC}-(X, Y)$ & $\mathrm{SC}-(T D, B P)$ \\
\cline { 2 - 3 } & $\mathrm{SC} ?(X, Y, Z)$ & $\mathrm{SC} ?(T D, M S, P C), \mathrm{SC} ?(A M, O T, T S)$, \\
& $\mathrm{SC} ?(P C, T S, S E)$
\end{tabular}

\subsection{Expert Rules}

Besides constraint rules defined in section 3.2, expert rules are used to prune the unreasonable branches of successor behaviors in simulation runs. Expert rules are prior to constraint rules when executed. With simulation advancing, expert rules are modifiable if needed. Expert rules are designed as follows.

1. Before simulation runs, there are individual variables and organizational variables. But state variables such as $S E$ and $S E$ ' are empty. The initial qualitative values and change directions of state variables are assigned according to following 
rules, where $Z \in\left\{P C, T S, S E^{\prime}\right\}, Z_{l} \in\{M S, A M$, $M E\}, Z_{2} \in\left\{T D, O T, B P^{\prime}\right\}$.

(a) If $q$ val $Z_{1} \neq$ qvalZ $_{2}$ And $\left|q v a l Z_{1}-q v a l Z_{2}\right|=1$, Then $q$ valZ $=\min \left(q v a l Z_{1}\right.$, qval $\left._{2}\right)$

(b) If $q$ val $Z_{1} \neq$ qvalZ $_{2}$ And $\mid q$ val $Z_{1}$-qvalZ $Z_{2} \mid / 2=$ $\left|q v a l Z_{1}-q v a l Z_{2}\right| / 2$, Then qvalZ $=\mid$ qval $_{1^{-}}$ $q$ valZ $Z_{2} / 2$

(c) If $q$ val $Z_{1} \neq$ qvalZ $Z_{2}$ And $\left|q v a l Z_{1}-q v a l Z_{2}\right| \neq 1$ And $\mid q$ val $Z_{1}$-qval $Z_{2}|/ 2 \neq|$ qval $Z_{1}$-qval $Z_{2} \mid / 2$, Then $q$ valZ $=\min \left(\right.$ qval $Z_{1}, q$ val $\left.Z_{2}\right)+1$

(d) If $q$ val $Z_{1}>=3$, Then qdirZ $=$ qdir $Z_{1}$

(e) If $q$ val $Z_{1}<3$, Then $q \operatorname{dir} Z=q \operatorname{dir} Z_{2}$

2. During the simulation runs, rules listed in Table 3 are performed.

Table 3: Action Rules in Simulation Runs

\begin{tabular}{|c|c|c|}
\hline $\begin{array}{c}\text { State } \\
\text { Vaiables }\end{array}$ & If & Then \\
\hline \multirow[t]{2}{*}{$P C$} & $\begin{array}{c}\text { qvalMS }>= \\
3\end{array}$ & $\mathrm{SC} ?(T D, M S, P C)=\mathrm{SC}+(M S, P C)$ \\
\hline & qvalMS $<3$ & $\mathrm{SC} ?(T D, M S, P C)=\mathrm{SC}-(T D, P C)$ \\
\hline \multirow[t]{2}{*}{$T S$} & $\begin{array}{c}\text { qvalAM }>= \\
3\end{array}$ & $\mathrm{SC} ?(A M, O T, T S)=\mathrm{SC}+(A M, T S)$ \\
\hline & qvalA $M<3$ & $\mathrm{SC} ?(A M, O T, T S)=\mathrm{SC}+(O T, T S)$ \\
\hline \multirow{3}{*}{$S E$} & $\begin{array}{c}\text { qvalPC }>= \\
3\end{array}$ & $\mathrm{SC} ?(P C, T S, S E)=\mathrm{SC}+(P C, S E)$ \\
\hline & qvalPC $<3$ & $\mathrm{SC} ?(P C, T S, S E)=\mathrm{SC}+(T S, S E)$ \\
\hline & Any & $q v a l S E=q v a l S E^{\prime}, q \operatorname{dir} S E=q \operatorname{dir} S E^{\prime}$ \\
\hline \multirow[t]{2}{*}{$B P$} & $\begin{array}{c}\text { qvalAS }>= \\
3\end{array}$ & $\mathrm{SC} ?(F S, A S, B P)=\mathrm{SC}-(A S, B P)$ \\
\hline & qvalAS $<3$ & $\mathrm{SC} ?(F S, A S, B P)=\mathrm{SC}+(F S, B P)$ \\
\hline$S E$ & Any & $\begin{array}{c}\mathrm{SC} ?\left(B P^{\prime}, M E, S E^{\prime}\right)=\mathrm{SC}+\left(B P^{\prime},\right. \\
\left.S E^{\prime}\right)\end{array}$ \\
\hline
\end{tabular}

3. When qualX increases to 5 or decrease to 1 , it will stop increasing or decreasing. $q \operatorname{valX}\left(t_{i}\right)$ and qval $X\left(t_{i+1}\right)$ are qualitative values of variable $X$ at time stage $t_{i}$ and $t_{i+1}$, respectively. $q \operatorname{dir} X / \mathrm{SC}$ is the constraint for variable $X$. Thus, expert rules about $q \operatorname{dir} X=\{2+, 2-\}$ are designed as follows.

(a) If $q \operatorname{valX}\left(t_{i}\right)=5$ And ( $q \operatorname{dir} X\left(t_{i+1}\right)=+$ or $q \operatorname{dir} X\left(t_{i}\right)$ $=2+)$ And $q \operatorname{dir} X / \mathrm{SC}=+$, Then $q \operatorname{val} X\left(t_{i+1}\right)=2+$

(b) If $q \operatorname{val} X\left(t_{i}\right)=5$ And $q \operatorname{dir} X\left(t_{i}\right)=2+$ And qdir $X / \mathrm{SC}=-$, Then $q \operatorname{val} X\left(t_{i+1}\right)=+$

(c) If $q \operatorname{val} X\left(t_{i}\right)=1$ And ( $q \operatorname{dir} X\left(t_{i}\right)=-$ or $q \operatorname{dir} X\left(t_{i}\right)=$ 2-) And $q \operatorname{dir} X / \mathrm{SC}=-$, Then $q \operatorname{val} X\left(t_{i+1}\right)=2$ -

(d) If $q$ valX $\left(t_{i}\right)=1$ And $q \operatorname{dir} X\left(t_{i}\right)=2$ - And $\operatorname{qdir} X / \mathrm{SC}=+$, Then $q \operatorname{val} X\left(t_{i+1}\right)=-$

\subsection{Algorithmic Steps}

Based on causality model illustrated in Figure 1 and simulation model built in section 3, algorithm of simulation is designed as following steps:

1. Let $k=1, s=1$.

2. Initialization. Give values to $K$ (simulation times), and $S$ (simulation stages). Initialize the value of individual variables. Set distribution parameters of contextual variables.

3. Take simulation run for the $k$-th time, and let $k=$ $k+1$. generate values of contextual variables randomly. Yield $S E$ ' from $M E, S E$ from $S E$ ', $B P$ from $S E, B P^{\prime}$ from $B P, P C$ from $T D$ and $M S, T S$ from $A M$ and $O T$.

4. Take simulation run at the $s$-th stage, and let $s=s$ + 1. According to constraint rules and expert rules, yield $S E$ ' from $B P^{\prime}$ and $M E, S E$ from $P C$ and $T S, B P^{\prime}$ from $A S$ and $F S$.

5. If $s=S$, then go to Step 6. Otherwise, let $s=s+1$ and go to Step 4.

6. If $k=K$, then ends simulation for one candidate. Otherwise, let $k=k+1$ and go to Step 3 .

Run the above simulation steps for every candidate and select the best one according to evaluation rules (see section 3.1).

\section{VALIDATION AND APPLICATION}

To validate the simulation method proposed above, an example is given here for application: when the former head of R\&D department leaves his post, a successor is needed to fill the vacant position. Since the post is very important for a company, it is necessary to select carefully the most optimal one from candidates applying for the post. It is supposed that four candidates, named a, b, c and d, have got through strict examinations and interviews and entered the final circle of selection. Since values of individual variables can be measured by means of standard questionnaires and structural interviews (see section 3.1), the data are listed in Table 4.

Table 4: The Measured Values of Individual Variables

\begin{tabular}{|c|c|c|c|c|}
\hline \multirow{2}{*}{ Individual Variable } & \multicolumn{4}{|c|}{ Candidate } \\
\cline { 2 - 5 } & $\mathrm{a}$ & $\mathrm{b}$ & $\mathrm{c}$ & $\mathrm{d}$ \\
\hline$M E$ & 5 & 5 & 5 & 5 \\
\hline$A S$ & 1 & 4 & 3 & 4 \\
\hline$A M$ & 5 & 5 & 1 & 3 \\
\hline$B P^{\prime}$ & 4 & 4 & 2 & 3 \\
\hline
\end{tabular}


Suppose contextual variables are subjected to uniform distribution within a certain scope. Following cases are designed to perform the validation and application.

- $\quad$ Case 1: As illustrated in Table 5, TD, $M S$ and $O T$ are around "middle". While $F S$ is always "highest".

- Case 2: As illustrated in Table 6, TD is "highest". $M S$ and FS are "highest" and sometimes "lowest". $O T$ is "middle".

- Case 3: As illustrated in Table 7, TD changes from "lowest" to "highest" gradually. $M S$ are "middle", FS and $O T$ are always "lowest".

- Case 4: As illustrated in Table 8, TD and $M S$ change between "highest" and "middle". FS and $O T$ are both "highest".

The simulation model and algorithm in section 3 is coded by Visual Basic 6.0. The number of simulation times $K$ and the number of simulation stages $S$ are specified as 100 and 5, respectively. The data in Table 4-8 are inputted to the coded simulation model. The simulation results are listed in Table 9-12.

Table 5: Contextual Variables in Case 1

\begin{tabular}{|c|c|c|c|c|}
\hline \multirow{2}{*}{ Stage } & \multicolumn{4}{|c|}{ Contextual Variables } \\
\cline { 2 - 5 } & $F S$ & $T D$ & $M S$ & $O T$ \\
\hline 1 & {$[0.75,1]$} & {$[0.35,0.6]$} & {$[0.35,0.6]$} & {$[0.35,0.6]$} \\
\hline 2 & {$[0.75,1]$} & {$[0.55,0.8]$} & {$[0.55,0.8]$} & {$[0.55,0.8]$} \\
\hline 3 & {$[0.75,1]$} & {$[0.35,0.6]$} & {$[0.35,0.6]$} & {$[0.35,0.6]$} \\
\hline 4 & {$[0.75,1]$} & {$[0.15,0.4]$} & {$[0.15,0.4]$} & {$[0.15,0.4]$} \\
\hline 5 & {$[0.75,1]$} & {$[0.35,0.6]$} & {$[0.35,0.6]$} & {$[0.35,0.6]$} \\
\hline
\end{tabular}

Table 6: Contextual Variables in Case 2

\begin{tabular}{|c|c|c|c|c|}
\hline \multirow{2}{*}{ Stage } & \multicolumn{4}{|c|}{ Contextual Variables } \\
\cline { 2 - 5 } & $F S$ & $T D$ & $M S$ & $O T$ \\
\hline 1 & {$[0.75,1]$} & {$[0.75,1]$} & {$[0.75,1]$} & {$[0.35,0.6]$} \\
\hline 2 & {$[0,0.2]$} & {$[0.75,1]$} & {$[0,0.2]$} & {$[0.35,0.6]$} \\
\hline 3 & {$[0.75,1]$} & {$[0.75,1]$} & {$[0.75,1]$} & {$[0.35,0.6]$} \\
\hline 4 & {$[0,0.2]$} & {$[0.75,1]$} & {$[0,0.2]$} & {$[0.35,0.6]$} \\
\hline 5 & {$[0.75,1]$} & {$[0.75,1]$} & {$[0.75,1]$} & {$[0.35,0.6]$} \\
\hline
\end{tabular}

Table 7: Contextual Variables in Case 3

\begin{tabular}{|c|c|c|c|c|}
\hline \multirow{2}{*}{$\begin{array}{c}\text { Stag } \\
\mathbf{e}\end{array}$} & \multicolumn{4}{|c|}{ Contextual Variables } \\
\cline { 2 - 5 } & $F S$ & $T D$ & $M S$ & $O T$ \\
\hline 1 & {$[0.75,1]$} & {$[0,0.2]$} & {$[0.35,0.6]$} & {$[0,0.2]$} \\
\hline 2 & {$[0.75,1]$} & {$[0.15,0.4]$} & {$[0.35,0.6]$} & {$[0,0.2]$} \\
\hline 3 & {$[0.75,1]$} & {$[0.35,0.6]$} & {$[0.35,0.6]$} & {$[0,0.2]$} \\
\hline 4 & {$[0.75,1]$} & {$[0.55,0.8]$} & {$[0.35,0.6]$} & {$[0,0.2]$} \\
\hline 5 & {$[0.75,1]$} & {$[0.75,1]$} & {$[0.35,0.6]$} & {$[0,0.2]$} \\
\hline
\end{tabular}

Table 8: Contextual Variables in Case 4

\begin{tabular}{|c|c|c|c|c|}
\hline \multirow{2}{*}{ Stage } & \multicolumn{4}{|c|}{ Contextual Variables } \\
\cline { 2 - 5 } & $F S$ & $T D$ & $M S$ & $O T$ \\
\hline 1 & {$[0.75,1]$} & {$[0.75,1]$} & {$[0.75,1]$} & {$[0.75,1]$} \\
\hline 2 & {$[0.75,1]$} & {$[0.55,0.8]$} & {$[0.55,0.8]$} & {$[0.75,1]$} \\
\hline 3 & {$[0.75,1]$} & {$[0.75,1]$} & {$[0.75,1]$} & {$[0.75,1]$} \\
\hline 4 & {$[0.75,1]$} & {$[0.55,0.8]$} & {$[0.55,0.8]$} & {$[0.75,1]$} \\
\hline 5 & {$[0.75,1]$} & {$[0.75,1]$} & {$[0.75,1]$} & {$[0.75,1]$} \\
\hline
\end{tabular}

Table 9: Simulation Results of Case 1

\begin{tabular}{|c|c|c|c|c|c|c|c|}
\hline \multirow{2}{*}{ Candidate } & \multicolumn{6}{|c|}{ Simulation Stage } & \multirow{2}{*}{ Probability } \\
\hline & 0 & 1 & 2 & 3 & 4 & 5 & \\
\hline A & $(4,0)$ & $(4,0)$ & $(4,+)$ & $(5,+)$ & $(5,+)$ & $(5,2+)$ & 0.08 \\
\hline $\mathrm{B}$ & $(4,0)$ & $(4,0)$ & $(4,+)$ & $(5,0)$ & $(5,-)$ & $(4,-)$ & 0.09 \\
\hline $\mathrm{C}$ & $(3,0)$ & $(3,0)$ & $(3,+)$ & $(4,0)$ & $(4,-)$ & $(3,-)$ & 0.09 \\
\hline $\mathrm{D}$ & $(4,0)$ & $(4,0)$ & $(4,+)$ & $(5,0)$ & $(5,-)$ & $(4,-)$ & 0.07 \\
\hline
\end{tabular}

Table 10: Simulation Results of Case 2

\begin{tabular}{|c|c|c|c|c|c|c|c|}
\hline \multirow{2}{*}{ Candidate } & \multicolumn{5}{|c|}{ Simulation Stage } & \multirow{2}{*}{ Probability } \\
\cline { 2 - 7 } & 0 & 1 & 2 & 3 & 4 & 5 & \\
\hline A & $(4,0)$ & $(4,0)$ & $(4,-)$ & $(3,-)$ & $(2,-)$ & $(1,+)$ & 0.07 \\
\hline B & $(4,0)$ & $(4,0)$ & $(4,-)$ & $(3,-)$ & $(2,-)$ & $(1,-)$ & 0.11 \\
\hline C & $(3,0)$ & $(3,0)$ & $(3,-)$ & $(2,-)$ & $(1,-)$ & $(1,-)$ & 0.11 \\
\hline D & $(4,0)$ & $(4,0)$ & $(4,-)$ & $(3,-)$ & $(2,-)$ & $(1,-)$ & 0.12 \\
\hline
\end{tabular}

Table 11: Simulation Results of Case 3

\begin{tabular}{|c|c|c|c|c|c|c|c|}
\hline \multirow{2}{*}{ Candidate } & \multicolumn{5}{|c|}{ Simulation Stage } & \multirow{2}{*}{ Probability } \\
\cline { 2 - 7 } & 0 & 1 & 2 & 3 & 4 & 5 & \\
\hline A & $(4,0)$ & $(4,0)$ & $(4,0)$ & $(4,+)$ & $(5,0)$ & $(5,+)$ & 0.18 \\
\hline B & $(4,0)$ & $(4,0)$ & $(4,0)$ & $(4,0)$ & $(4,+)$ & $(5,+)$ & 0.15 \\
\hline C & $(3,0)$ & $(3,0)$ & $(3,0)$ & $(3,+)$ & $(4,+)$ & $(5,+)$ & 0.28 \\
\hline D & $(4,0)$ & $(4,0)$ & $(4,0)$ & $(4,+)$ & $(5,0)$ & $(5,+)$ & 0.18 \\
\hline
\end{tabular}

Table 12: Simulation Results of Case 4

\begin{tabular}{|c|c|c|c|c|c|c|c|}
\hline \multirow{2}{*}{ Candidate } & \multicolumn{6}{|c|}{ Simulation Stage } & \multirow{2}{*}{ Probability } \\
\hline & 0 & 1 & 2 & 3 & 4 & 5 & \\
\hline A & $(4,0)$ & $(4,0)$ & $(4,-)$ & $(3,-)$ & $(2,-)$ & $(1,+)$ & 0.13 \\
\hline B & $(4,0)$ & $(4,0)$ & $(4,-)$ & $(3,+)$ & $(4,+)$ & $(5,+)$ & 0.09 \\
\hline $\mathrm{C}$ & $(3,0)$ & $(3,0)$ & $(3,-)$ & $(2,0)$ & $(2,-)$ & $(1,-)$ & 0.09 \\
\hline $\mathrm{D}$ & $(4,0)$ & $(4,0)$ & $(4,-)$ & $(3,-)$ & $(2,-)$ & $(1,-)$ & 0.11 \\
\hline
\end{tabular}

The analysis of the results is as follows.

- Analysis of Case 1: As illustrated in Table 9, $S E$ of candidate (a), (b), (c) and (d) increase at the beginning. But after stage $3, S E$ of candidate (a) 
keeps increasing, while $S E$ of (b), (c) and (d) start decreasing. It indicates that, keeping high $F S$ and modifying $M S$ and $O T$ according to $T D$ can lead to $S E$ increasing. This is consistent with the common sense. Therefore, candidate (a) is the best one according to the highest value rule (see section 3.1). Candidate (b) and (c) have not much difference according to the highest probability rule. But according to the combination rule, candidate (b) is the best one.

- Analysis of Case 2: As illustrated in Table 10, the $S E$ of candidate (a), (b), (c) and (d) keep decreased all the time. But $S E$ of candidate (a) has a increase at last stage. This indicates that, keeping $F S$ and $T D$ stable and keeping $M S$ and $O T$ unstable will lead $S E$ to be decreased. This is consistent with the common sense. Therefore, candidate (a) is still the best one according to the highest value rule (see section 3.1). Candidate (b) and (c) have not yet much difference according to the highest probability rule. But according to the combination rule, candidate (c) is the best one.

- Analysis of Case 3: As illustrated in Table 11, candidate (a) is the best one according to the highest value rule (see section 3.1). Candidate (c) is the best one according to the combination rule.

- Analysis of Case 4: As illustrated in Table 12, candidate (b) is the best one according to the highest value rule (see section 3.1). While candidate (a) is the best one according to the combination rule.

The above analysis shows that the results of simulation runs are consistent with the common senses. The best one selected is different according to the different preferences and the needs of decision-makers. Thus, we think that the proposed simulation model is valid. Through the above simulation runs and analysis, it also indicates that the simulation model can aid decision-maker of the enterprise to select the optimal manager of a department.

\section{CONCLUSION}

The following conclusions can be drawn from the above works:

1. Based on theory of self-efficacy and its researches in the field of human resource management, a causality model is proposed.

2. Based on the causality model, a qualitative simulation model is proposed by modifying QSIM. Its algorithm steps are designed. This work comprises the following propositions:

(a) the generating-filtering pattern of QSIM is modified into constraint-generating-filtering pattern by integrating with the probability of quantitative simulation.

(b) The state transition rules are simplified. The transition between time point and time interval in QSIM are avoided. While the state transition is designed between time points (i.e. time stages).

(c) Constraint rules are expanded. When two variables exert effect on the third variable, the transition rule are designed.

(d) Expert rules and probability are designed to make the filter more effective.

3. The simulation model is coded by Visual Basic 6.0. It is used to validate the model through an example.

Because the theory of self-efficacy is derived from social psychology, managerial self-efficacy is the application of self-efficacy in the management field, which involves various subjective factors. The causality model in this paper is proposed considering the primary factors. On the other hand, QSIM is improved referring the quantitative information(i.e., probability). But it leads to new problems such as the rationality of expert rules, its combination with constraint rules, etc. Therefore, the causality model of managerial self-efficacy will be improved to provide more detailed information for selection decision. QSIM will be improved to fit for properties in the management field in further studies.

\section{ACKNOWLEDGMENTS}

This work has taken place in the Qualitative Simulation Team Work for Complex Management Systems at Institute for Intelligent Management \& System Simulation, School of Management, Huazhong University of Science and Technology. It is supported by the National Natural Science Foundation of China (Grant No. 70271029) and the Natural Science Foundation of Hubei Province, China (Grant No. 2004ABA069). The authors would like to thank the anonymous referees for their helpful comments and suggestions on early versions of this paper.

\section{REFERENCES}

Bandura, A., and R. E. Wood. 1989. Effect of perceived controllability and performance standards on selfregulation of complex decision making. Journal of Personality and Social Psychology, 56(5):805-814.

Bandura, A. Self efficacy: The exercise of control. New York, NY: W. H. Freeman \& Company.

Cervone, D., N. Jiwani, and R. E. Wood. 1991. Goal setting and the differential influence of self-regulatory processes on complex decision-making performance. 
Journal of Personality and Social Psychology, 61(2):257-266.

Clancy, D. J., and B. J. Kuipers. 1998. Qualitative Simulation as a Temporally-extended Constraint Satisfaction Problem. Proceedings of the 15th National Conference on Artificial Intelligence (AAAI-98), Cambridge, Ma: AAAI/MIT Press.

Earley, P. C., C. B. Gibson, and C. C. Chen. 1999. "How did I do?" versus "How did we do?" : Cultural contrasts of performance feedback use and self-efficacy. Journal of Cross-Cultural Psychology, 30(5):594-619.

Hu, B., and G. Xia. 2003. Group Behavior Simulation Oriented Knowledge Description and its Reasoning Algorithm. Proceedings of the International Conference on Management Science \& Engineering, Georgia, USA, August 15-17.

$\mathrm{Hu}$, B., and G. Xia. 2005. Integrated Qualitative Simulation Method for Group Behavior. Journal of Artificial Societies and Social Simulation, 8(2). Available via <http://jass.soc.surrey.sc.uk/8/2/1 . html>.

Kuipers, B. J. 1993. Reasoning with qualitative model. Artificial Intelligence, 59:125-132.

Lauschruger, H. K. S., and J. Shamian. 1994. Staff nurses' and nurses managers' perceptions of job-related empowerment and managerial self-efficacy. Journal of Nursing Administration, 24:38-47.

Robertson, I. T., and G. Sadri. 1993. Managerial selfefficacy and managerial performance. British Journal of Management, 4:37-45.

Wood, R. E., and A. Bandura. 1989. Impact of conceptions of ability on self-regulatory mechanisms and complex decision making. Journal of Personality and Social Psychology, 56(3):407-415.

\section{AUTHOR BIOGRAPHIES}

HU BIN, Ph.D., professor, director of Institute for Intelligent Management \& System Simulation, School of Management, Huazhong University of Science and Technology, P.R. China. He received the B.S. and M.S. in Wuhan University of Technology, P.R. China (1989 and 1994). He received the Ph.D. in Management Science and Engineering from Huazhong University of Science and Technology, P.R. China (1999). His research interests include Management System Simulation, Decision Support System and Artificial Intelligence. His research papers have published in many Chinese journals and international journals. His email address is <bin_hu@mail. hust. edu. cn>.

DONG SHENGPING, doctorate student of Institute for Intelligent Management \& System Simulation, School of Management, Huazhong University of Science and Technology, P.R. China. He received the B.S. in Wuhan University of Technology, P.R. China in 2002, and M.S. in
Huazhong University of Science and Technology, P.R. China in 2005. His research interests include Management System Simulation, Decision Support System and Artificial Intelligence. His research papers have published in many Chinese journals. His e-mail address is $<$ dsp.phdagmail. com>. 